\title{
Was meinen wir mit Biometrie?
}

Vor einigen Wochen bekam ich eine E-Mail von einer Institution, die gerne biometrische Gesichtserkennung einsetzen möchte und Rat sucht: Welche Verfahren denn in Betracht kämen, wenn eine Fehlerrate von höchstens 5\% akzeptabel sei - so war die Anfrage formuliert. Auf eine unscharfe Frage kann man keine hilfreiche Antwort geben. So war es zunächst notwendig zu klären, ob es sich um eine biometrische Verifikations- oder IdentifikationsAnwendung handelt und ob mit der genannten Toleranz die in einem Technologietest unter Verwendung von Testdatenbanken gemessenen Algorithmenfehler oder die in einem Szenariotest gemessenen Systemfehler einschließlich Datenerfassungsfehler zu verstehen seien. Bei einer allgemeinen Anfrage kann man im Dialog mehr oder weniger leicht ergründen, was denn nun wirklich mit der Anfrage gemeint war. Schwieriger und mitunter auch folgenschwerer wird ein unscharf formulierter Text in einer Ausschreibung, wenn in der Folge Anbieter einer vermeintlich vergleichbaren Technologie die Leistungskriterien des gesuchten Systems anders auslegen, als der spätere Betreiber des biometrischen Systems. Ein Rechtsstreit ist dann absehbar und eigentlich nicht $\mathrm{zu}$ entscheiden, wenn offensichtlich aneinander vorbei geredet wurde. Die klare und eindeutige Definition der Begriffe, die zur Festlegung von Leistungskriterien benutzt werden, ist daher aus vertragsjuristischen und letztlich wirtschaftlichen Gründen erstrebenswert. Seit Anfang des Jahres 2013 steht nun die Internationale Norm ${ }^{1}$ zur Verfügung, die von der Working Group 1 im Sub-Committee 37 des ISO/IEC JTC1 über die letzten 10 Jahre erarbeitet wurde. Diese Norm definiert Begriffe wie die false match rate (FMR), die false non-match rate (FNMR) oder die failure to acquire rate (FTA) eindeutig, so dass das oben genannte Problem vermieden werden kann. Auch die Beschreibung biometrischer Prozesse wird durch die konsequente Verwendung des standardisierten Vokabulars ${ }^{2}$ einfacher und klarer. Ein Beispiel ist der mitunter zu lesende Satz „In der Gesichtserkennung werden die biometrischen Merkmale des Gesichts erfasst und mit einer Merkmalsextraktion Merkmale berechnet, die in einem Template gespeichert werden“. Das ist verwirrend: Quelle des Prozesses und Ergebnis des Prozesses werden gleichermaßen als Merkmale bezeichnet? Klarheit schafft in diesem Fall die Verwendung des harmonisierten Vokabulars, so dass der Satz umformuliert werden kann in „In der Gesichtserkennung werden die biomet-

1 ISO/IEC 2382-37 „Information Technology - Vocabulary - Part 37: Biometrics"

2 Das standardisierte Harmonized Biometric Vocabulary (ISO/IEC 2382-37) steht mit einer deutschen Übersetzung online zur Verfügung unter:

http://www.christoph-busch.de/standards.html rischen Charakteristika des Gesichts erfasst und mit einer Merkmalsextraktion Merkmale berechnet, die in einem Template gespeichert werden". Damit wird zwischen Quelle (Charakteristikum) und Ergebnis (Merkmale) klar unterschieden. Auch der absurde aber tatsächlich schon verwendete Satz „A false non-match occurs if two matched samples are matched and found not to match" kann durch Anwendung des ISO/IEC Standards widerspruchsfrei und besser formuliert werden und ergibt dann „A false nonmatch occurs if two samples from the same source are compared and found not to match".

Neben einer unmissverständlichen Sprache ist ein gemeinsames solides biometrisches Wissen bei Entscheidern aber auch bei Betreibern und Anwendern von biometrischen Systemen hilfreich, um die Leistungsfähigkeiten und Möglichkeiten der Biometrie richtig einschätzen zu können. Im November 2011 wurde daher die European Association for Biometrics ${ }^{3}$ (EAB) gegründet. Als Non-Profit-Organisation verfolgt sie das Ziel, die nutzbringende Verwendung von biometrischen Systemen zu unterstützen und auf der EAB-Plattform unabhängige Informationen zu biometrischen Verfahren und Anwendungen zur Verfügung zu stellen. Mit bereits hundert Mitgliedern hat sich in kurzer Zeit ein Kompetenznetzwerk etabliert, dass einerseits die thematische Vielfalt der biometrischen Modalitäten und Anwendungen abdeckt und andererseits durch ein Netzwerk von nationalen Kontaktstellen die geographische Breite Europas abdeckt. Zudem werden die existierenden nationalen Fachgruppen, wie etwa die im TeleTrusT Bundesverband IT-Sicherheit e.V. etablierte Arbeitsgruppe Biometrie, mit ihren Aktivitäten in die Europäische Arbeit der EAB-Dachorganisation eingebunden. Durch die Ausschreibung des European Research and Industry Awards wird die industrielle und akademische Forschung von Nachwuchswissenschaftlern gefördert. Christian Rathgeb war Preisträger dieses Forschungspreises im vergangenen Jahr und berichtet in diesem Heft in einer Kurzfassung über die mit dem Preis ausgezeichnete Arbeit.

Die EAB ist ein gutes Beispiel dafür, dass Europäisches Denken und Handeln sinnvoll ist. Europaweite Diskussion von Positionen durch eine elektronische Plattform und in Veranstaltungen von Mitgliedern und Nicht-Mitgliedern führt zu einem stärkeren Bekenntnis zu den für alle geltenden Regeln und Prozessen.

3 Informationen zur EAB sind zu finden unter: http://www.eab.org 